\title{
¿BINARIO O NO BINARIO? MORFOLOGÍA DE GÉNERO EN ESPAÑOL: DIFERENCIAS DEPENDIENTES DE LA TAREA
}

\author{
Gabriela Mariel ZUNINO \& Noelia Ayelén STETIE*1
}

\section{RESUMEN}

Existe evidencia empírica en distintas lenguas sobre cómo la computación de la morfología de género durante el procesamiento psicolingüístico incide en la conformación de representaciones sexo-genéricas. Sin embargo, no existe evidencia empírica sobre el procesamiento de variantes morfológicas no binarias en español (-x o -e) en contraste con la variante de masculino genérico (-o). Para analizar este fenómeno, realizamos dos experimentos: una tarea de juicios de aceptabilidad y una de comprensión de oraciones para evaluar procesamiento online. Los resultados muestran diferencias dependientes de la tarea, es decir, de los procesos subyacentes que se ponen en juego en cada una. En los juicios de aceptabilidad, que involucran procesos estratégicos mediados por las creencias y la norma lingüística, el masculino genérico resulta más aceptable para referir a grupos mixtos. En la tarea de comprensión de oraciones, que indaga sobre procesos automáticos y representaciones implícitas, las formas no binarias provocaron consistentemente una referencia hacia grupos mixtos y los tiempos de respuesta indicaron que estas variantes morfológicas no conllevan un costo de procesamiento mayor que el masculino genérico.

PALABRAS CLAVE: Psicolingüística. Género. Morfología. Estereotıpos de género.

\section{Introducción}

La pregunta por las múltiples formas en que puede influir el lenguaje en el pensamiento data de al menos un siglo (SAPIR, 1921; ZLATEV; BLOMBERG, 2015). A través de los años, y desde distintos enfoques -teóricos y empíricos-, este problema ha ido tomando diferentes perfiles. Formas extremas de esta idea, que sostenían un determinismo lingüístico, más que una influencia o sesgo, han provocado reacciones opuestas también extremas (PINKER, 1999), que desatienden matices o propuestas intermedias, también denominadas "débiles" (EVERETT, 2013; LUCY, 1996; SAPIR, 1921; SCOTTO; PÉREZ, 2020; WHORF, 1956; ZLATEV; BLOMBERG, 2015). Inicialmente, la hipótesis de relativismo lingüístico sostenía que la lengua que hablamos moldea nuestro pensamiento. Es decir, que debido a las diferentes categorías y

\footnotetext{
${ }^{1}$ Universidad de Buenos Aires (UBA) - Consejo Nacional de Investigaciones Científicas y Técnicas (CONICET), Instituto de Lingüística (FFyL- UBA), Buenos Aires, Argentina, gmzunino@conicet.gov.ar
} 
distinciones -sobre todo semánticas- que realiza cada lengua, sus hablantes se ven condicionades $^{2}$ a prestar atención a distintos aspectos del entorno y eso les lleva, en última instancia, a generar representaciones diferentes sobre los mismos eventos del mundo. Descartar un determinismo fuerte no supone eliminar la posibilidad de analizar las múltiples y complejas formas en las que se da la relación entre pensamiento y lenguaje, o cognición, representaciones mentales y formas lingüísticas. Existen, ciertamente, diversas y muy actuales propuestas en torno a este punto (EVERETT, 2013; SCOTTO; PÉREZ 2020; ZLATEV; BLOMBERG, 2015): el lenguaje como "potenciador" del pensamiento, el lenguaje como "intromisión" u “obstáculo", el lenguaje como "focalizador", el lenguaje como "inductor" y algunas variantes de la conocida hipótesis de "pensar para hablar" (SLOBIN, 1991; 1996). Varias de estas perspectivas han sido retomadas por estudios empíricos en el marco de la psicolingüística experimental y han logrado recolectar evidencia de respaldo para sustentar algunos modos de sesgo o influencia de las formas lingüísticas sobre la cognición. En este marco se encuadran muchos de los estudios sobre la proyección de las marcas morfológicas de género de las distintas lenguas hacia las representaciones sexo-genéricas que manipulan les hablantes de dichas lenguas; trabajos que intentan indagar sobre los posibles sesgos de género que las formas lingüísticas propias de la e rructura de una le gua podrían acarrear para la cognición.

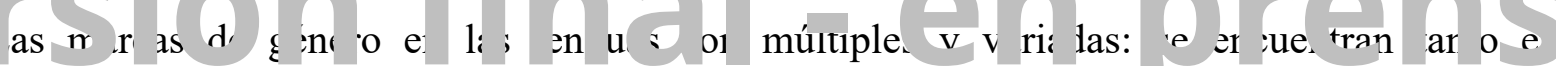
características o elementos gramaticales como en aspectos léxicos, y más allá de la estructura de las lenguas, los usos discursivos de las formas lingüísticas y las maneras en las que se organizan las construcciones también pueden desplegar diversos sesgos de género (LEAPER 2014; PÉREZ; MORAGAS, 2020; STAHLBERG et al., 2007). Así, el género gramatical es sólo una de las múltiples dimensiones en las que podemos analizar la relación entre representaciones sexo-genéricas, construcción de sentidos vinculados a relaciones e identidades de género y (usos de) la lengua.

En este sentido, es preciso comenzar por aclarar que las lenguas difieren respecto de cómo marcan el género gramatical. A lo largo de los años se han propuesto diferentes taxonomías (CORBETT, 1991; DIXON, 1987; LEAPER, 2014; PREWITT-FREILINO; CASWELL; LAAKSO, 2012). La más reciente es la propuesta por Gygax et al. (2019) que considera cinco

\footnotetext{
${ }^{2}$ En este trabajo hemos decidido utilizar la forma morfológica con [-e] para referir a personas de cualquier género. Creemos que, como parte de una comunidad lingüística, pero también como parte de una comunidad académica que está transitando debates políticos y transformaciones sociales potentes en relación con los sesgos de género no sólo en la lengua sino también en los modos de construcción y circulación de conocimiento, esta decisión aporta a la reflexión y el gesto performativo vuelca a las prácticas concretas del ámbito científico discusiones actuales y profundas sobre las innumerables proyecciones de un ordenamiento heteropatriarcal en las distintas dimensiones de la vida pública y privada de las personas.
} 
tipos de lenguas: lenguas con género gramatical, lenguas con género natural, lenguas con combinación de género gramatical y natural, y lenguas sin género -con algunos rastros de género gramatical y sin rastros-. Dentro del primer grupo, en donde se encuentra el español y lenguas como el francés, polaco y alemán, el género controla la concordancia gramatical y todos los sustantivos -aquellos que refieren a entidades animadas tanto como inanimadas- tienen género asignado. Por ejemplo, en español flexionamos en género la mayoría de los nombres de rol, que refieren a personas, como secretaria y secretario o enfermero y enfermera, y también asignamos género gramatical a objetos inanimados, como la leche y el cartón.

Uno de los fenómenos estudiados, por ejemplo, es si para les hablantes de lenguas con marcas de género obligatorias y paradigmas de género binarios el sesgo de interpretación genérica deja de ser tan arbitrario como suponen los estudios gramaticales y pasa a proyectar representaciones asociadas a las identidades sexo-genéricas de personas a otras palabras que refieren a entidades no humanas (EVERETT, 2013; FLAHERTY, 2001; KONISHI 1993; SAALBACH; IMAI; SCHALK, 2012; SEGEL; BORODITSKY, 2011; SERA et al., 2002) .

Otro de los puntos más profusamente estudiados es el caso de los llamados nombres de rol, y dado que estos se instancian de forma diferente en cada lengua, dependiendo del paradigma de género gramaícal (GYG × et al 2019), urge el interrogante de si dichas realizaciones iferer e g ne ar di inta: re re en $x$ or s nentales. (en c ra pala $r$, rom tire $]$ ar ead por Scotto y Pérez (2020), hasta qué punto la influencia del género gramatical de las lenguas sobre la cognición se puede analizar en el marco de la hipótesis del relativismo lingüístico.

Experimentos en varias lenguas (KAUFMANN; BOHNER, 2014; LEAPER, 2014; SATO; GYGAX; GABRIEL, 2016; SCZESNY; MOSER; WOOD, 2015; VIGLIOCCO et al., 2005) han mostrado evidencia empírica de sesgos estables - no contingentes o coyunturales en usos lingüísticos particulares en contextos comunicativos puntuales- de ciertas formas lingüísticas en la cognición, es decir, en las representaciones mentales que manipulamos durante tareas tanto mediadas como no mediadas por la lengua.

Un fenómeno central de esta línea de estudios es hasta qué punto el llamado masculino genérico funciona como tal, es decir, marca y sirve para representar grupos de personas con género no uniforme. En un paradigma binario como el del español es clara la asimetría entre los usos de la morfología de femenino para referir a grupos exclusivos de mujeres -algunes lo mencionan como un ejemplo más de que el femenino es la forma marcada: JIMÉNEZ RODRIGO; ONSALO; TRAVERSO CORTÉS, 2011; KONISHI, 1993; PREWITT-FREILINO; CASWELL; LAAKSO, 2012; STAHLBERG et al., 2007-respecto de los usos ambiguos del masculino para referir tanto a conjuntos de varones como de personas con identidad de género 
no uniforme (BRAUN; SCZESNY; STAHLBERG, 2005; CACCIARI; PADOVANI, 2007; GYGAX; GABRIEL, 2008, GYGAX et al., 2008, MACIUSZEK; POLAK; SWIATKOWSKA, 2019; MISERSKY; MAJID; SNIJDERS, 2019).

Algunos estudios empíricos en español muestran que el sesgo generado por el masculino genérico es identificable y que parece tener un efecto sostenido en la cognición. Por ejemplo, Kaufmann y Bohner (2014), con un estudio pionero en español, analizan la forma binaria "con barra" (los/as) y dos innovaciones morfológicas para generar formas inclusivas no binarias (la -@ y la -x). En su experimento, les participantes debían leer historias breves y generar un completamiento de fragmentos de palabras. Si bien encontraron un sesgo débil en los completamientos modulado por el género de cada participante, no hallaron diferencias debidas a la forma lingüística utilizada en los ítems.

En el presente trabajo, desarrollamos la primera etapa de una línea de investigación más amplia que intenta analizar de qué modo les hablantes de español procesamos formas morfológicas binarias y variantes no binarias como modos de representar y referir a grupos de personas con identidad de género no uniforme. En ese sentido, este estudio dialoga con otros que indagan sobre las proyecciones cognitivas del masculino genérico, pero le suma otro fenómeno escasamente estudiado: li comparación cc el procesamiento psicolingüístico de las dos

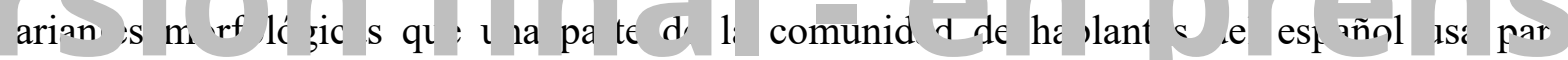
flexionar sustantivos que refieran a personas $-\mathrm{y}$ todas las clases de palabras que deban concordar en género con ellos- bajo un paradigma de género no binario. Bajo la expresión "formas no binarias", nos referimos específicamente a las variantes morfológicas que también son conocidas como "lenguaje inclusivo". Mientras que las niñas se utiliza para referir a grupos de mujeres y los niños a grupos de varones, las innovaciones morfológicas como lxs niñxs o les niñes se utilizan para referir a grupos sin género uniforme. La denominación misma puede ser discutida, y, de hecho, lo es. Para evitar interpretaciones posibles en las que "inclusivo" supone sostener una norma binaria como eje para sólo aplicar una tercera forma como modo de "abarcar" lo que se supone queda por fuera de un binarismo hegemónico, y para no reducir la noción de "formas inclusivas del uso de la lengua" a la morfología de género de esa lengua, usaremos el término formas (morfológicas) no binarias.

Así, nos proponemos analizar: 1. si el masculino genérico en español proyecta representaciones mentales genéricas o, en cambio, condiciona esas representaciones con un sesgo hacia la referencia exclusiva de varones; 2. si les hablantes de español aceptan las tres variantes morfológicas en la misma medida; 3. si las variantes morfológicas no binarias (-x y -e), adoptadas en español como variantes morfológicas inclusivas, logran representar 
adecuadamente grupos no uniformes en su identidad de género, y 4. si el costo de procesamiento para realizar esa referencia durante la comprensión de oraciones que incluyan sintagmas con formas no binarias es mayor que en el caso de la forma con masculino genérico.

\section{Experimento 1}

Como primer acercamiento al estudio del fenómeno, diseñamos una tarea de juicios de aceptabilidad de sintagmas nominales en plural. Esto nos permitiría analizar la forma en que les hablantes juzgan la aceptabilidad de las frases en función de dos factores: el nivel de estereotipicidad de los nombres de rol implicados y el tipo de morfología utilizada -formas binarias y no binarias para referir a grupos de personas con identidad sexo-genérica no uniforme-. Como toda tarea de juicios, este experimento indaga sobre un proceso estratégico, consciente y mediado por creencias, que puede exhibir sesgos culturales de distinto tipo. Pero nos interesaba especialmente, como primer paso en esta investigación, contar con una medida de este tipo. Además, esta tarea nos permite verificar si los niveles de estereotipicidad considerados a priori en e aiseño se provec an efectivamente como factor determinante para

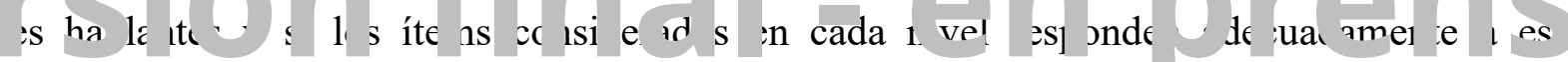
clasificación.

Nuestra hipótesis central sostiene que los juicios de aceptabilidad variarán en función del nivel de Estereotipicidad de los sintagmas nominales según el siguiente patrón: por un lado, esperamos que, en el caso de los nombres de rol con estereotipicidad baja -los niños-, el masculino genérico produzca una referencia más consistente hacia un grupo de personas mixto; para el caso de la estereotipicidad alta -los plomeros-, sin embargo, sería menos probable la interpretación de dicho sintagma nominal como el adecuado para referenciar a un grupo que no estuviera compuesto íntegramente por varones. Además, nuestras predicciones suponían que las formas no binarias tendrían más aceptación para los términos de estereotipicidad baja -les niñes- que para los de estereotipicidad media y alta -les plomeres-, por ser, los primeros, sintagmas que se utilizan con más frecuencia flexionados con formas no binarias.

\section{Participantes}


Participaron 75 personas, 53 mujeres (edad: $M=32.55, D E=11.04$; rango=18 y 62 ) y 22 varones (edad: $M=35.68 ; D E=11.03$; rango=20 y 60). Del total de les participantes, 12 indicaron haber terminado el secundario, 31 estar cursando una carrera de nivel superior, 29 haberse recibido de una carrera de nivel superior y 3 contar con estudios de posgrado. Todes les participantes son hablantes de español como lengua primera y residentes en Argentina.

\section{Materiales}

Los ítems lingüísticos utilizados presentaban sintagmas nominales simples (determinante + sustantivo) en plural, que referían a grupos de personas. Para la elaboración de las frases se consideraron dos variables independientes con tres niveles para cada una. Por un lado, la morfología utilizada como genérico: genérico masculino (-o) y las dos formas no binarias (-x y -e). Por otro lado, la estereotipicidad de los nombres de rol respecto de su adscripción a uno u otro género, en un sistema sexo-genérico binario: baja, media y alta.

Se eligieron 6 ítems para cada nivel de estereotipicidad y se presentaron en las tres variantes morfológicas tue en españ t podrían referir c grupos de personas sin género uniforme. es decir.

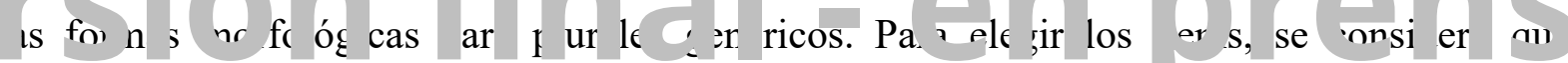
admitieran morfemas en femenino y en masculino, es decir, se evitaron nombres de rol que no presentaran variación en género -como estudiantes-. También se tuvo expreso cuidado de no seleccionar palabras que requirieran de cambios ortográficos al utilizar la forma no binaria [-e] -como amigue-. Además, se diseñaron 6 ítems más en femenino que funcionaron como distractores. Las frases se organizaron en 3 listas contrabalanceadas de 21 ítems cada una. A continuación se presentan ejemplos de los ítems utilizados según el nivel de Estereotipicidad:

Estereotipicidad baja: Los hijos/Lxs hijxs/Les hijes

Estereotipicidad media: Los funcionarios/Lxs funcionarixs/Les funcionaries

Estereotipicidad alta: Los plomeros/Lxs plomerxs/Les plomeres

\section{Procedimiento}

La tarea fue diseñada y tomada mediante un formulario de Google que asignaba aleatoriamente una lista diferente a cada participante. En todos los casos, se presentó primero un consentimiento informado que debía ser aceptado para acceder a las preguntas demográficas y 
al experimento. Se les pidió a les participantes que indicaran identidad de género, máximo nivel de estudios alcanzado y edad. Luego se presentó la consigna: se les pidió que juzgaran el grado de aceptabilidad de cada frase en una escala de 1 a 7 , siendo 1 una aceptabilidad muy baja y 7 muy alta. Se hizo especial hincapié en que el juicio debía llevarse a cabo sobre la base de la comprensión de esa frase como una forma válida para referirse a un conjunto de personas sobre el que no quiere hacerse distinción de género, es decir, para referirse a grupos de personas de género no uniforme, o grupos mixtos.

La tarea se distribuyó por redes sociales a potenciales participantes que hablaran español rioplatense, particularmente que habitaran en el AMBA (Área Metropolitana de Buenos Aires). La participación fue voluntaria y les participantes no recibieron ninguna remuneración a cambio.

\section{Resultados}

El análisis de los datos se llevó a cabo a partir de las puntuaciones de la escala Likert de cada particinante para cada sint Ima nominal Lo datos fueron procesados mediante el programa $R$ ersion 4 1. e li in $\operatorname{rrfaz} R, u$ io $\mathrm{R}$ C $\mathrm{R}$ : TEAM, (1) 1 ) y s util og or los racu tes Ime (BATES; MAECHLER; BOLKER; WALKER, 2015), lmer Test (KUZNETSOVA; BROCKHOFF; CHRISTENSEN, 2017) y MuMIn (BARTON, 2020) para realizar los análisis estadísticos.

Dadas las discusiones teóricas sobre la mejor manera de tratar estadísticamente datos surgidos de escalas, siguiendo a Endresen y Janda (2015), desarrollamos múltiples análisis: ANOVA, Regresión Lineal Múltiple, Modelo Mixto y Chi cuadrado. En todos los casos el cuadro de resultados es similar y los contrastes estadísticamente significativos fueron los mismos.

En este caso, optamos por reportar los resultados del Modelo Lineal Mixto (LMM) ${ }^{3}$. Cada nivel de los factores fijos fue codificado como -0.5 y 0.5 , compatible con lo que se denomina suma escalada (SCHAD; VASISHTH; HOHENSTEIN; KLIEGL, 2020). La Morfología y la Estereotipicidad se colocaron como efectos fijos y con interacción y se anidó a estas la Identidad de género de les participantes. Participantes e Ítems se colocaron como efectos aleatorios cruzados. Los resultados del modelo se muestran en la Tabla 1.

\footnotetext{
${ }^{3}$ En todos los análisis se intentó primero el modelo máximo (BARR; LEVY; SCHEEPERS; TILY, 2013). Si el modelo no convergía, se eliminaban los efectos aleatorios que representaban la menor varianza en el modelo máximo no convergente hasta que se alcanzaba la convergencia.
} 
Como se observa en las Figuras 1 y 2, tanto para los varones como para las mujeres, el patrón muestra una escala de aceptación: las frases correspondientes a nombres de rol de estereotipicidad alta tienen una menor aceptación para todas las formas no binarias, especialmente la variante morfológica con [-e] y especialmente entre los varones.

Figura 1 - Medias juicios de aceptabilidad por Figura 2 - Aceptación alta por Estereotipicidad, Estereotipicidad, Morfología e Identidad de Morfología e Identidad de género
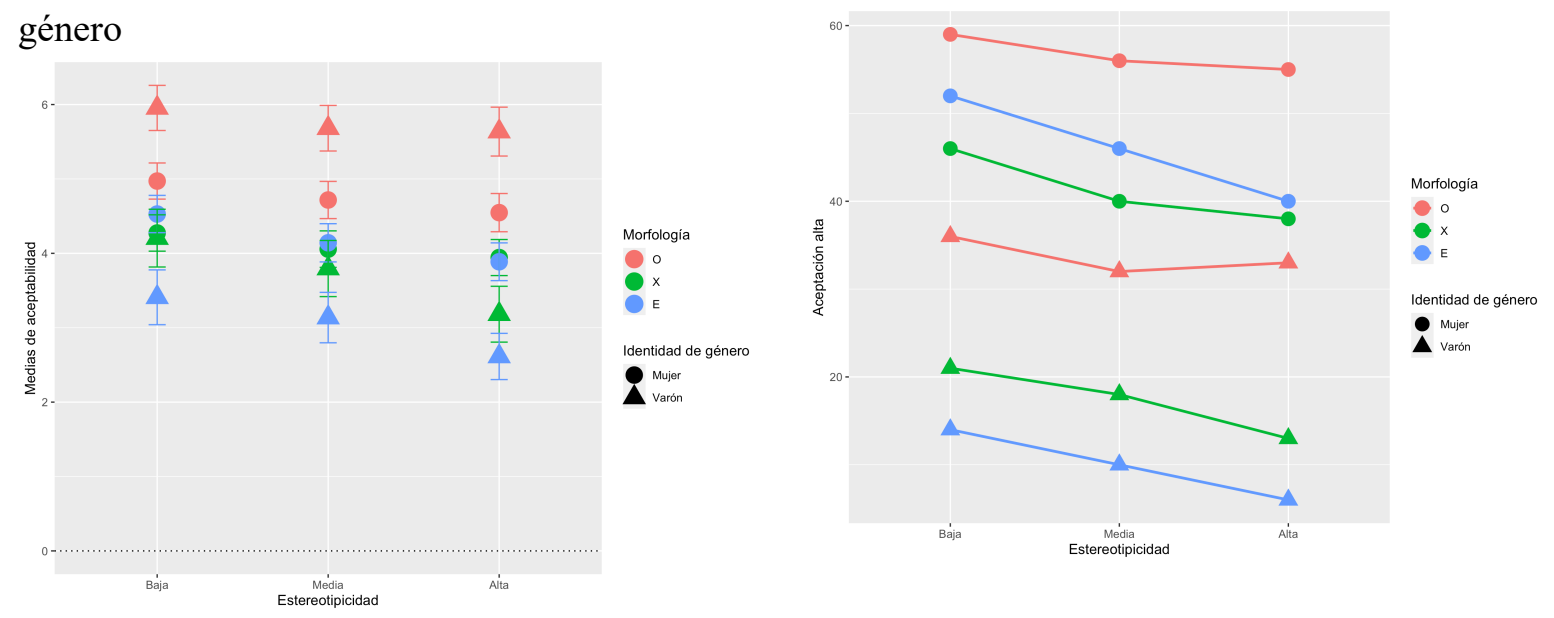

Tabla 1 - Reporte del LMM

Observacione total $^{-}=1350$

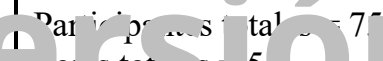

I liel s tota. $=5$.

Efectos fijos

\begin{tabular}{|c|c|c|c|c|}
\hline & Est/Beta & Error estándar & $t$ & $p$ \\
\hline Intercepto & 4.26014 & 0.13792 & 30.890 & $<2 \mathrm{e}-16 * * *$ \\
\hline Morfología_X-O & -1.34236 & 0.50168 & -2.676 & $0.009134 * *$ \\
\hline Morfología_E-X & -0.29003 & 0.23681 & -1.225 & 0.224388 \\
\hline Estereotipicidad_Media-Baja & -0.30024 & 0.08119 & -3.698 & $0.000499 * * *$ \\
\hline Estereotipicidad_Alta-Media & -0.28250 & 0.08119 & -3.480 & $0.000984 * * *$ \\
\hline Morfología_X-O:Estereotipicidad_Media-Baja & -0.05174 & 0.19887 & -0.260 & 0.795702 \\
\hline Morfología_E-X:Estereotipicidad_Media-Baja & -0.02245 & 0.19887 & -0.113 & 0.910526 \\
\hline Morfología_X-O:Estereotipicidad_Alta-Media & -0.26626 & 0.19887 & -1.339 & 0.186067 \\
\hline
\end{tabular}




\begin{tabular}{|c|c|c|c|c|}
\hline Morfología_E-X:Estereotipicidad_Alta-Media & -0.02375 & 0.19887 & -0.119 & 0.905359 \\
\hline Morf_O:Estereotipicidad_Baja:Id.género_V-M & 0.94961 & 0.59054 & 1.608 & 0.111531 \\
\hline Morf_X:Estereotipicidad_Baja:Id.género_V-M & -0.07836 & 0.60409 & -0.130 & 0.897103 \\
\hline Morf_E:Estereotipicidad_Baja:Id.género_V-M & -1.11293 & 0.61087 & -1.822 & 0.072013 \\
\hline Morf_O:Estereotipicidad_Media:Id.género_V-M & 0.95802 & 0.59054 & 1.622 & 0.108441 \\
\hline Morf_X:Estereotipicidad_Media:Id.género_V-M & -0.25577 & 0.60409 & -0.423 & 0.673077 \\
\hline Morf_E:Estereotipicidad_Media:Id.género_V-M & -1.01188 & 0.61087 & -1.656 & 0.101340 \\
\hline Morf_O:Estereotipicidad_Alta:Id.género_V-M & 1.13346 & 0.59054 & 1.919 & 0.058289 \\
\hline Morf_X:Estereotipicidad_Alta:Id.género_V-M & -0.75579 & 0.60409 & -1.251 & 0.214344 \\
\hline Morf_E:Estereotipicidad_Alta:Id.género_V-M & -1.27199 & 0.61087 & -2.082 & $0.040345 *$ \\
\hline \multicolumn{5}{|c|}{ Efectos aleatorios } \\
\hline $1+\infty$ & \multicolumn{2}{|c|}{ wian: } & 8 & \\
\hline Participantes (Intercepto) & \multicolumn{2}{|c|}{1.11446} & \multicolumn{2}{|c|}{1.0557} \\
\hline Participantes (Morfología_X-O) & \multicolumn{2}{|c|}{15.24133} & \multicolumn{2}{|c|}{3.9040} \\
\hline Participantes (Morfología_E-X) & \multicolumn{2}{|c|}{3.07716} & \multicolumn{2}{|c|}{1.7542} \\
\hline Ítems (Intercepto) & \multicolumn{2}{|c|}{0.01243} & \multicolumn{2}{|c|}{0.1115} \\
\hline \multicolumn{5}{|c|}{ Ajuste del modelo } \\
\hline \multirow[t]{2}{*}{$\mathrm{R}^{2}$} & \multicolumn{2}{|c|}{ Marginal } & \multicolumn{2}{|c|}{ Condicional } \\
\hline & \multicolumn{2}{|c|}{0.0769889} & \multicolumn{2}{|c|}{0.8538611} \\
\hline \multicolumn{5}{|c|}{$\begin{array}{l}\text { Los } p \text {-valor de los efectos fijos fueron calculados con las aproximaciones de Satterthwaite. } \\
\text { Ecuación del modelo: Aceptabilidad } \sim(\text { Morfología * Estereotipicidad) / Identidad de género }+(1+\text { Morfología } \mid \\
\text { Participantes })+(1 \mid \text { Ítems })\end{array}$} \\
\hline
\end{tabular}

Como se observa en la Tabla 1, se exhibe un efecto principal del factor Estereotipicidad (MediaBaja: $p=0.00049$; Alta-Media: $p=0.000984)$. En cuanto a las variantes de genérico, se 
encontraron diferencias estadísticamente significativas entre la forma de masculino genérico (o) y ambas formas no binarias $(p=0.009134)$, pero no entre las dos variantes no binarias $(p=0.224388)$. No se encontraron interacciones entre la Morfología y la Estereotipicidad ${ }^{4}$. En relación con la variable Identidad de género, se encontró un efecto anidado a la estereotipicidad alta y la variante no binaria [-e] $(p=0.040345)$ : las mujeres presentaban mayor aceptación que los varones.

\section{Discusión}

En primer lugar, nos interesaba utilizar esta tarea como estudio normativo para verificar los niveles de Estereotipicidad considerados a priori en nuestro diseño experimental. De acuerdo con los resultados obtenidos, los ítems incluidos en cada nivel de este factor parecen ser adecuados.

En segundo lugar, como esperábamos, el factor Morfología mostró un efecto estadísticamente significativo: las formas no binarias fueron menos aceptadas que el masculino genérico. Aunaue ros rrupos de vi ones y muieres estaban desequilibrados v no hubo diferencias

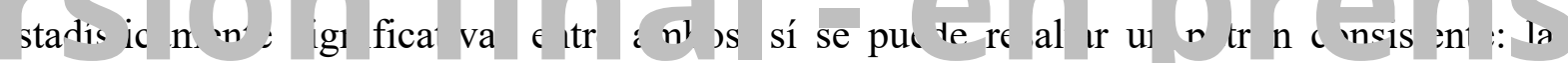

formas no binarias son menos aceptadas entre los varones que entre las mujeres, en particular la variante morfológica con [-e], la única que presentó diferencias estadísticamente significativas en los casos de alta estereotipicidad.

Por último, no se encontraron interacciones estadísticamente significativas entre la Estereotipicidad y la Morfología. Sin embargo, los resultados mostraron un patrón claro: 1. los nombres de rol con alta estereotipicidad-como los plomeros-fueron menos aceptados cuando se presentaban con formas no binarias -especialmente [-e]-; 2. el masculino genérico fue más aceptado transversalmente para los tres niveles de Estereotipicidad; 3. el nivel medio de estereotipicidad parece ser el grupo con resultados menos consistentes (ver Figuras 1 y 2 ). Una explicación posible de estos resultados es que el uso más frecuente de formas no binarias genere mayor aceptación y que, efectivamente, las palabras que categorizamos como de alta estereotipicidad no suelen utilizarse en sus variantes morfológicas no binarias y aquellas que clasificamos como estereotipicidad media sí se utilizan más frecuentemente con formas no binarias.

\footnotetext{
${ }^{4}$ Además, corrimos un modelo en el que anidamos el factor Estereotipicidad a la Morfología y encontramos diferencias estadísticamente significativas para las formas no binarias, no para el masculino genérico.
} 
Las tareas de juicios de aceptabilidad suponen procesos estratégicos condicionados por ideologías lingüísticas, paradigmas normativos y representaciones sociales de género. En esta línea, como adelantamos en nuestras hipótesis, podemos decir que cuando la tarea involucra procesos estratégicos fuertemente mediados por las creencias y la norma lingüística, el masculino genérico resulta aceptable como forma para referir a grupos mixtos, por encima de las formas no binarias. Si bien los juicios de aceptabilidad resultan datos de gran relevancia, en tanto también exhiben las disposiciones conscientes de les hablantes en relación con las formas lingüísticas y sus usos sociales, posiblemente no permitan verificar qué ocurre cuando los procesos mentales subyacentes implican manipular representaciones sexo-genéricas de modo implícito y computar elementos lingüísticos de manera automática durante la lectura.

Nuestros resultados muestran que cuando les hablantes deben juzgar aceptabilidad, en tanto proceso consciente, voluntario y estratégico, los juicios se corresponden con el sostenimiento de formas binarias y se condicen con la norma que supone que el masculino genérico en español logra referir a grupos de personas sin sesgo de identidad sexo-genérica. Para analizar si esta tendencia se mantiene durante procesos mentales automáticos y que requieran manipular representaciones sexo-genéricas implícitas, y en función de poder evaluar el procesamiento psicolinguístico de estas fo mas morfológica de modo online. $\mathrm{v}$ no sólo a través de paradigmas

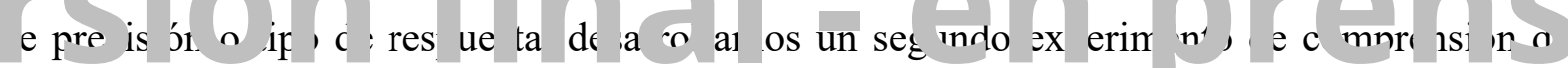
oraciones.

\section{Experimento 2}

El segundo experimento fue diseñado a partir de la clasificación de Estereotipicidad de sintagmas nominales evaluada en el Experimento 1. Se buscaba analizar el procesamiento psicolingüístico online durante la comprensión de oraciones que incluyeran dichos sintagmas; y se esperaba verificar cuáles eran las representaciones sexo-genéricas implícitas que les hablantes construían como referencia para esos sintagmas durante el proceso de comprensión. Diseñamos una tarea de comprensión de oraciones que supone la lectura a ritmo propio de una oración, para luego presentar una pregunta sobre la referencia posible del sintagma nominal en posición de sujeto. La respuesta a dicha pregunta se hacía a través de un paradigma de opciones múltiples.

Las hipótesis centrales que planteamos para el Experimento 2 suponen que: 1 . habrá un efecto del factor Estereotipicidad de los nombres de rol para la selección de posibles referentes; 2. 
existirá una interacción entre Estereotipicidad y Morfología que se volcará no sólo en el tipo de opción elegida sino también en el tiempo que toma hacer la elección; 3. si bien la selección de referentes de las formas no binarias será más consistente hacia grupos con género no uniforme, los tiempos para hacer esa elección pueden mostrar una ventaja para la forma de masculino genérico, como forma no marcada en español.

\section{Participantes}

En esta tarea participaron 551 personas de las cuales hubo que remover 13 (menores de 18 años o participantes que no declararon su edad). De las restantes (538), 386 eran mujeres (edad: $M=34.52 ; D E=11.60 ;$ rango=19 y 98), 131 varones (edad: $M=34.15 ; D E=12.09 ;$ rango=18 y 82) y 21 personas se identificaron como no cisgénero ${ }^{5}$ (edad: $M=29.67 ; D E=8.21$; rango=19 y 55). Del total de les participantes, 399 declararon vivir en el Área Metropolitana de Buenos Aires y 139 fuera de esta área ${ }^{6}$. En cuanto a la escolaridad, 34 participantes declararon haber completado el secundario, 103 estar cursando una carrera de educación superior o tener estudios incomnleios, $\checkmark 401$ haber $t$ mmnado una carr ra de nivel superior o tener estudios de posgrado.

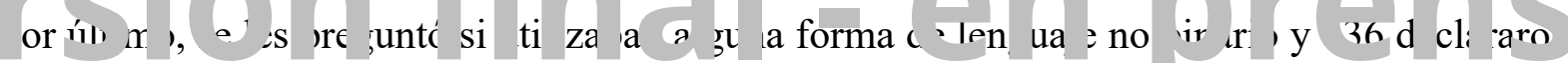
que no, 111 que lo utilizaban poco u ocasionalmente y 291 que lo utilizaban con frecuencia.

\section{Materiales}

A partir de los sintagmas nominales utilizados en la tarea anterior, se elaboraron oraciones con dichas frases como sujeto gramatical. Se mantuvo el mismo diseño experimental y se elaboraron 18 oraciones que se presentaron en las tres variantes morfológicas. A continuación se presentan ejemplos para cada condición de Estereotipicidad:

Baja estereotipicidad: Los/xs/es maestros/xs/es usan recursos variados durante la alfabetización inicial.

Media estereotipicidad: Los/xs/es enfermeros/xs/es tienen obligación de actuar si hay un accidente en la vía pública.

\footnotetext{
${ }^{5}$ El grupo de personas que agrupamos bajo esta denominación estaba compuesto por: no binaries, chica no binaria, varón trans, gay cis, ninguno, agénero, género fluido, lesbiana, demi-chica, marica.

${ }^{6}$ Esto incluye diversas provincias de Argentina.
} 
Alta estereotipicidad: Los/xs/es plomeros/xs/es con matrícula pueden hacer trabajos en edificios y consorcios.

Para cada oración se armó una pregunta de opciones múltiples sobre la comprensión de la frase nominal, a fin de indicar si esta se refería a un grupo de mujeres, de varones o mixto. Además, se sumaron tres opciones más de respuesta que operaban como distractores. Para ello, se utilizaron nombres que, en Argentina, suelen utilizarse con alta frecuencia para nombrar a mujeres o a varones. A continuación se presenta una pregunta de ejemplo y sus opciones de respuesta:

¿A cuál de las siguientes opciones puede referir "los maestros”?

a. Carolina.

b. Manuel.

c. Manuel, Marta y otras personas.

d. Carolina, Marta y otras mujeres.

e. Manuel, Federico y otros varones.

f. Ninguna de las opciones anteriores.

Específicamente lo que nos interesaba medir era la distinción entre las opciones c. y e. en el ejemnlo anteiror, es decir nitre la opción q e refería a un gruno de personas mixto y la aue efería a un cr aj nt de ar ne. 1 or er de aparic on d l; op in es va aha e nod aleatorio en cada ítem.

Además, a partir de los fillers utilizados en la tarea anterior, se elaboraron 18 oraciones con los sintagmas nominales en femenino, a las que se sumaron otros 12 distractores que utilizaban las tres variantes de genérico, pero que contenían una pregunta de comprensión diferente. De este modo, se equilibró la exposición a oraciones y preguntas diversas, en virtud de no generar aprendizaje o entrenamiento dentro de la tarea. Por ejemplo:

Lxs pintorxs prefieren trabajar con óleos para conseguir mejores texturas y relieves.

¿Qué prefieren lxs pintorxs?

a. Trabajar con óleos.

b. Pintar con acuarelas.

c. Usar muchos colores.

d. Usar pinceles anchos.

e. Realizar trazos finos.

f. Ninguna de las opciones anteriores. 
Los estímulos se dividieron en tres listas contrabalanceadas en las que se incluyeron 2 ítems de cada condición. Cada lista estaba compuesta por 48 ítems: 18 experimentales y 30 fillers -los mismos para las tres listas-.

\section{Procedimiento}

La tarea fue diseñada y tomada mediante el software PCIbex (ZEHR; SCHWARZ, 2018), que asignaba aleatoriamente una lista diferente a cada participante. En todos los casos, se presentó primero un consentimiento informado que debía ser aceptado para acceder a las preguntas sobre datos demográficos y al experimento. Se les pidió a les participantes que indicaran identidad de género, máximo nivel de estudios alcanzado, edad, nacionalidad, ciudad de residencia y con qué frecuencia utilizaban alguna forma morfológica no binaria. Luego se presentó la consigna: se les indicó que primero leyeran las oraciones y que luego respondieran una pregunta de opciones múltiples lo más rápido posible y basándose en su primera impresión. La oración se presentaba completa en una primera pantalla en letras negras sobre fondo blanco. La siguiente pantalla mostraba la preg nta y las oncion $\mathrm{s}$, a las que les narticinantes debían resnonder

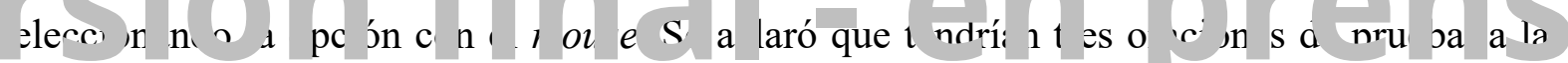

que le seguían tres oraciones más de práctica, que para les participantes ya eran parte de la tarea experimental.

El experimento se difundió por redes sociales entre hablantes de español, preferentemente, variedad rioplatense. La participación fue voluntaria y les participantes no recibieron ninguna remuneración a cambio.

\section{Resultados}

Análisis de los tipos de respuesta

Los datos fueron analizados con los mismos programas y paquetes que el Experimento 1. Para el análisis de los tiempos y el tipo de respuesta se tomaron las correspondientes a los ítems respondidos adecuadamente. Es decir, se consideraron sólo las respuestas que referían a un conjunto de varones o a un grupo mixto de personas, y se descartaron aquellas que referían a una sola persona, a un grupo de mujeres o a ninguno. De las respuestas eliminadas, ninguna 
refería a una sola persona -ni mediante un nombre típicamente femenino ni uno típicamente masculino-, 51 referían a un grupo de mujeres y 342 referían a "ninguna de las opciones"7 . Los ítems descartados por respuestas incorrectas representan el $4.06 \%$ de los datos.

Además, otro factor a tener en cuenta en el análisis de los datos es que, debido al desbalanceo de la muestra en cuanto a les participantes no cisgénero -recordemos que representaban el $3,81 \%-$, se optó por removerles para la realización de los modelos estadísticos. Sin embargo, decidimos sostener estos datos en los gráficos, con el fin de comenzar con un esbozo de las diferencias en este grupo, para continuar su análisis en futuros trabajos.

Como se puede ver en la Figura 3 y en la Tabla 2, ambas variantes morfológicas no binarias ($\mathrm{x}$ y -e) generan consistentemente una representación y referencia inequívoca hacia grupos mixtos de personas, independientemente del nivel de Estereotipicidad del nombre de rol. Sin embargo, no sucede lo mismo con el masculino genérico. En primer lugar, parecería no funcionar inequívocamente como genérico. En segundo lugar, la representación y referencia que se construyen parecerían depender del nivel de Estereotipicidad: aquellos nombres con estereotipicidad baja -como los niños o los maestros- generan más representaciones efectivamente genéricas, mientras que los de estereotipicidad alta-como los plomeros o los herreros-geheran represe ciciones eminent nente masculinas

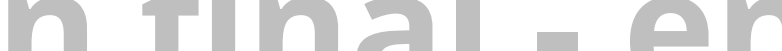

Figura 3 - Tipos de respuesta por Estereotipicidad y Morfología

\footnotetext{
${ }^{7}$ Un análisis más detallado de aquellas respuestas marcadas como "ninguna" devela que esta opción se utilizó mayoritariamente para responder a las oraciones en lenguaje no binario - 162 respuestas a sintagmas con la variante [-e] y 158 a sintagmas con la variante [-x]-. Consideramos que se trató de una forma de señalar que las formas de lenguaje no binario no pueden ser entendidas, ya que de estas 320 respuestas, 279 pertenecían a personas que habían declarado no usar ninguna forma de lenguaje no binario. Además, de las 342 respuestas, 221 (64,62\%) referían a personas identificadas como mujeres, $116(33,92 \%)$ a personas identificadas como varones y $5(1,46 \%)$ referían a una persona identificada como no cisgénero. De las restantes, consideramos que se trató de un error por falta de atención. Se evaluó la posibilidad de que, con esas respuestas, les participantes hayan querido señalar que la forma no binaria no refiere sólo a varones y mujeres, sino también a personas no cisgénero, pero luego se descartó debido a que en la opción que refería a un grupo mixto de personas se incluyeron nombres estereotípicamente utilizados para mujeres, otros utilizados para varones y la aclaración "y otras personas".
} 


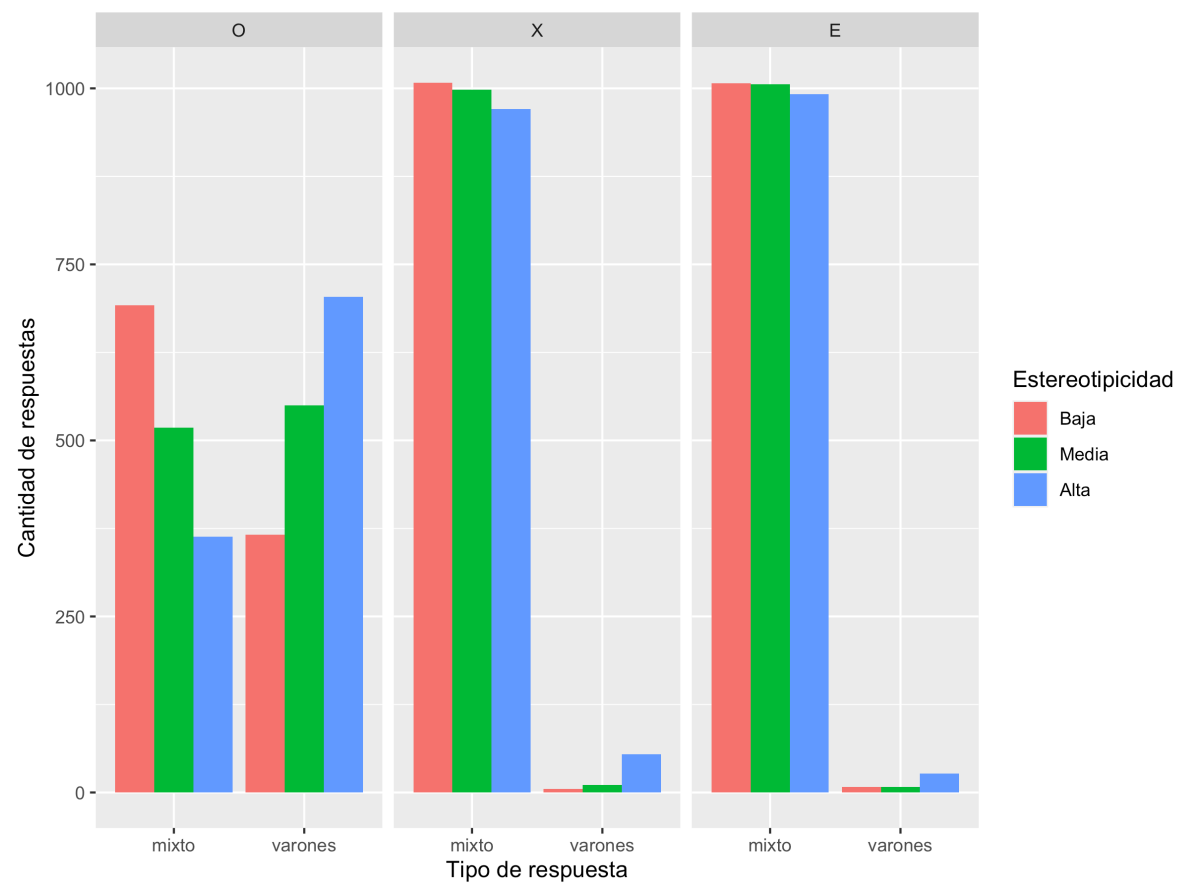

También, como se puede ver en la Tabla 3, estas representaciones varían según la identidad de género de les participantes. Por un lado, aunque los porcentajes de respuesta de mujeres y varones ror ura u otra opcín éan en la misma dirección, entre las mujeres es aún más marcada

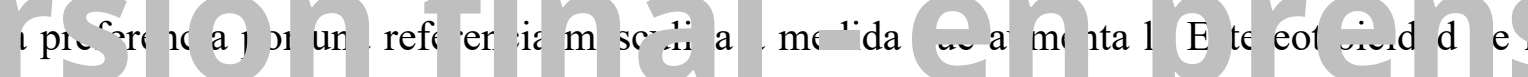
nombres de rol. Por otro lado, para les participantes identificades comc no cisgénero, la variante [-o] no parecería generar representaciones efectivamente genéricas aún cuando la estereotipicidad de los nombres de rol sea baja.

Tabla 2 - Porcentajes de tipos de respuesta por condición

\begin{tabular}{|l|l|l|l|l|l|l|}
\hline \multirow{2}{*}{} & \multicolumn{5}{|c|}{ Estereotipicidad } \\
\cline { 2 - 7 } & \multicolumn{2}{|c|}{ Baja } & \multicolumn{2}{c|}{ Media } & \multicolumn{2}{c|}{ Alta } \\
\hline Tipo de respuesta & Mixto & Varones & Mixto & Varones & Mixto & Varones \\
\hline Morfología-O & $65,41 \%$ & $34,59 \%$ & $48,50 \%$ & $51,50 \%$ & $34,02 \%$ & $65,98 \%$ \\
\hline Morfología-X & $99,51 \%$ & $0,49 \%$ & $98,91 \%$ & $1,09 \%$ & $94,73 \%$ & $5,27 \%$ \\
\hline Morfología-E & $99,21 \%$ & $0,79 \%$ & $99,21 \%$ & $0,79 \%$ & $97,35 \%$ & $2,65 \%$ \\
\hline
\end{tabular}


Tabla 3 - Porcentajes de tipos de respuesta para variante morfológica [-o]

\begin{tabular}{|l|l|l|l|l|l|l|}
\hline \multirow{2}{*}{} & \multicolumn{3}{|c|}{ Estereotipicidad } & \multicolumn{2}{c|}{ Alta } \\
\cline { 2 - 8 } & \multicolumn{2}{|c|}{ Baja } & \multicolumn{2}{c|}{ Media } & Mixto & Varones \\
\hline Tipo de respuesta & Mixto & Varones & Mixto & Varones & Mix & \\
\hline Identidad de género-Mujer & $63,95 \%$ & $36,05 \%$ & $45,96 \%$ & $54,04 \%$ & $30,12 \%$ & $69,88 \%$ \\
\hline Identidad de género-Varón & $74,61 \%$ & $25,39 \%$ & $59,85 \%$ & $40,15 \%$ & $48,26 \%$ & $51,74 \%$ \\
\hline Identidad de género-No cis & $35,71 \%$ & $64,29 \%$ & $24,39 \%$ & $75,61 \%$ & $17,07 \%$ & $82,93 \%$ \\
\hline
\end{tabular}

Debido a las características de los sintagmas en lenguaje no binario y a las diferencias en los tipos de respuesta presentadas en las Tablas 2 y 3 , los análisis estadísticos se realizaron, por un lado, sobre las respuestas mixtas y, por otro lado, sobre las respuestas de masculino genérico, las únicas que efectivamente habilitaban una respuesta por un conjunto de varones.

Como se observa en la T ivia 2, hubo unc diferencia estadísticamente significativa en las

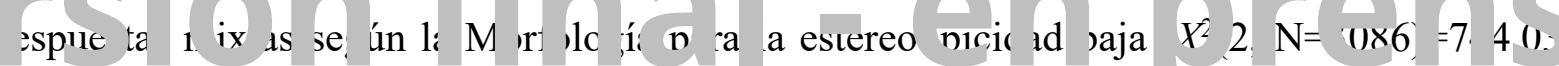
$p=<2.2 \mathrm{e}-16)$, media $\left(X^{2}(2, \mathrm{~N}=3091)=1189.6, p=<2.2 \mathrm{e}-16\right)$ y alta $\left(X^{2}(2, \mathrm{~N}=3111)=1431\right.$, $p=<2.2 \mathrm{e}-16)$. En los tres casos, las diferencias fueron entre el masculino genérico y ambas formas no binarias (-x y -e).

Para el análisis de las respuestas del masculino genérico, se realizó un GLM (Modelo Linear Generalizado) ya que se trataba de una variable binaria -respuesta por grupos mixtos o conjunto de varones-. Los porcentajes de la Tabla 3 sugieren que no había interacción entre la Identidad de género y la Estereotipicidad; sin embargo, al igual que en el caso de la correlación entre Identidad de género y Frecuencia de uso, este análisis requeriría ajustar un modelo con más factores de los que podemos desarrollar en este trabajo. En la Tabla 4 se presentan los resultados del modelo estadístico elegido para explicar los datos obtenidos.

Tabla 4 - Reporte del GLM

Observaciones totales $=3069$ (correspondientes a la variante $-\mathrm{o})$

Participantes totales $=516$

Ítems totales $=18$ 


\begin{tabular}{|c|c|c|c|c|}
\hline & Est/Beta & Error estándar & $z$ & $p$ \\
\hline Intercepto & -0.4348 & 0.2425 & -1.794 & 0.0729 \\
\hline Estereotipicidad_Media-Baja & 1.8924 & 0.3377 & 5.604 & $2.10 \mathrm{e}-08 * * *$ \\
\hline Estereotipicidad_Alta-Media & 1.5980 & 0.3334 & 4.793 & $1.64 \mathrm{e}-06 * * *$ \\
\hline Identidad de género_Varones-Mujeres & -1.7189 & 0.4284 & -4.012 & $6.01 \mathrm{e}-05 * * *$ \\
\hline \multicolumn{5}{|c|}{ Efectos aleatorios } \\
\hline & \multicolumn{2}{|c|}{ Varianza } & \multicolumn{2}{|c|}{$\mathrm{DE}$} \\
\hline Participantes (Intercepto) & \multicolumn{2}{|c|}{13.0154} & \multicolumn{2}{|c|}{3.6077} \\
\hline Ítems (Intercepto) & \multicolumn{2}{|c|}{0.2582} & \multicolumn{2}{|c|}{0.5082} \\
\hline \multicolumn{5}{|c|}{ Ajuste del modelo } \\
\hline $0 \quad z$ & \multicolumn{2}{|c|}{ Marginal } & \multicolumn{2}{|c|}{ Condicional } \\
\hline & \multicolumn{2}{|c|}{$0^{12513}$} & \multicolumn{2}{|c|}{$0.8 \cong 8$} \\
\hline
\end{tabular}

Como se desprende de la Tabla 4, se encontraron dos efectos principales: uno vinculado al factor Estereotipicidad y otro vinculado a la Identidad de género de les participantes. Estos efectos confirman las diferencias que se vislumbran en la Tabla 3. La diferencia entre respuestas por un grupo mixto o un conjunto de varones exhibe un efecto principal del factor Estereotipicidad (Media-Baja: $p=2.10 \mathrm{e}-08$; Alta-Media: $p=1.64 \mathrm{e}-06$ ). Además, los datos muestran un efecto de Identidad de género $(p=6.01 \mathrm{e}-05)$.

\section{Análisis de los tiempos}

Para el análisis de los tiempos de respuesta, se realizó una identificación de outliers y posterior transformación. Todas aquellas medidas que se encontraban a más de dos y medio desvíos estándar de la media por condición fueron reemplazadas por la media de cada participante en 
cada condición. Esto implicó reemplazar 245 datos, es decir el 2,64\% de la muestra (BAAYEN; MILIN, 2010; COUSINEAU; CHARTIER, 2010; RATCLIFF, 1993).

Además, antes de realizar los análisis estadísticos, se graficaron los tiempos de respuesta por condición para poder identificar visualmente si había algún patrón general en los datos. En la Figura 4 y la Tabla 5, se muestran los tiempos de reacción según el tipo de respuesta, la Morfología, la Estereotipicidad de los nombres de rol y la Identidad de género de les participantes $^{8}$. En cuanto a las respuestas que referían a un grupo mixto de personas, el patrón de tiempo entre las mujeres y los varones es similar, mientras que el patrón de les participantes identificades como no cisgénero es distinto. En relación con las respuestas que referían a un grupo de varones, les participantes no cisgénero no indicaron en ningún caso que los sintagmas con la variante no binaria [-e] podrían referir a este tipo de respuestas.

Figura 4 - Tiempos de respuesta según Tipo de respuesta, Identidad de género, Estereotipicidad y Morfología
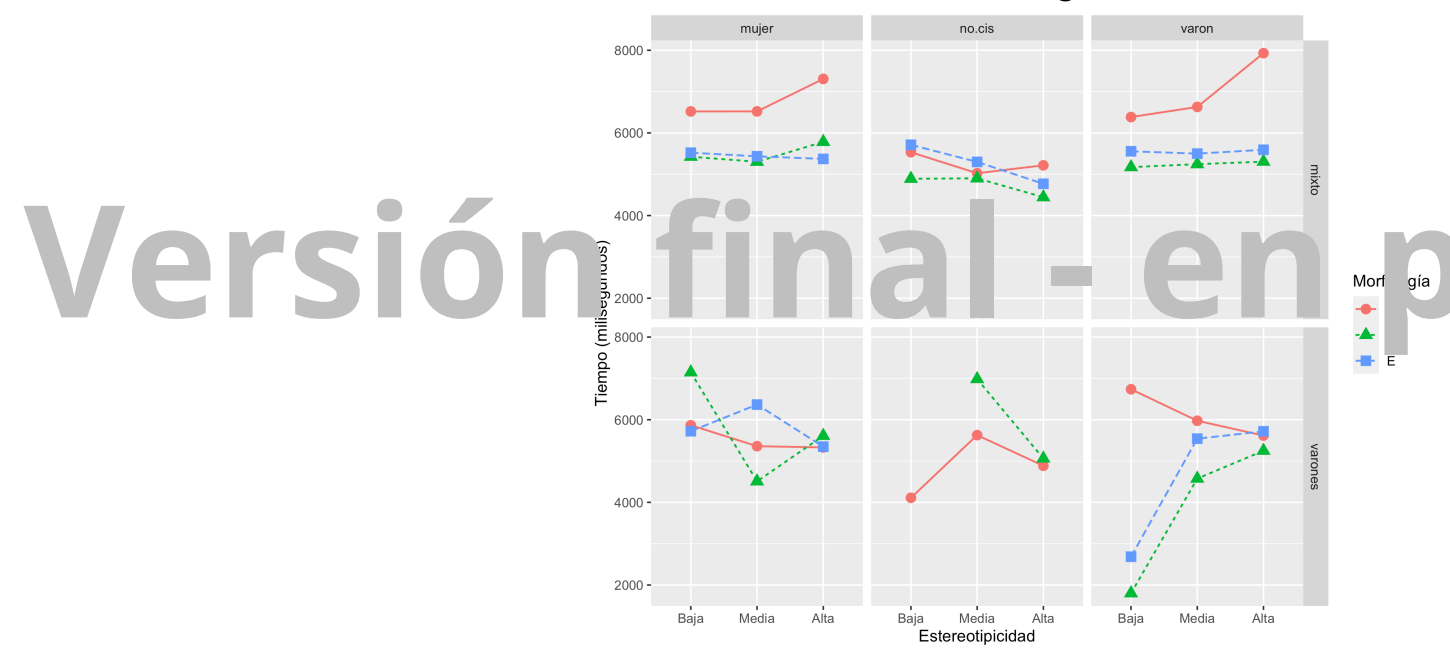

Tabla 5 - Medias y desvíos de los tiempos de respuesta por Identidad de género, Tipo de respuesta, Estereotipicidad y Morfología

\begin{tabular}{|l|c|c|c|c|c|c|}
\hline \multicolumn{3}{|c|}{ Mujeres } \\
\hline RTA & \multicolumn{3}{|c|}{ Mixto } & \multicolumn{3}{c|}{ Varones } \\
\hline Est. & Baja & Media & Alta & Baja & Media & Alta \\
\hline
\end{tabular}

\footnotetext{
${ }^{8}$ En un análisis complementario, estudiamos la relación entre el factor Identidad de género y la Frecuencia de uso espontáneo de formas no binarias reportada por les participantes. Surge de allí una correlación entre estas variables, debido a la complejidad de este análisis y las restricciones de espacio, decidimos desarrollar este punto en otro trabajo (Zunino \& Stetie, en evaluación).
} 


\begin{tabular}{|c|c|c|c|c|c|c|}
\hline $\mathrm{TR}$ & $M(D E)$ & $M(D E)$ & $M(D E)$ & $M(D E)$ & $M(D E)$ & $M(D E)$ \\
\hline$-\mathrm{O}$ & $6520(3558)$ & $6520(3818)$ & $7306(4166)$ & $5867(3274)$ & $5359(3482)$ & $5329(3302)$ \\
\hline$-X$ & $5424(2430)$ & 5301 (2439) & $5782(3022)$ & 7147 (3591) & 4509 (2080) & $5609(3750)$ \\
\hline$-E$ & $5521(2494)$ & $5434(2495)$ & $5371(2426)$ & $5722(2963)$ & $6365(4646)$ & $5348(2646)$ \\
\hline \multicolumn{7}{|c|}{ Varones } \\
\hline RTA & \multicolumn{3}{|c|}{ Mixto } & \multicolumn{3}{|c|}{ Varones } \\
\hline Est. & Baja & Media & Alta & Baja & Media & Alta \\
\hline $\mathrm{TR}$ & $M(D E)$ & $M(D E)$ & $M(D E)$ & $M(D E)$ & $M(D E)$ & $M(D E)$ \\
\hline$-\mathrm{O}$ & $6383(3073)$ & $6628(3423)$ & $7930(5233)$ & 6737 (4169) & $5975(3558)$ & $5616(4486)$ \\
\hline$-X$ & $5172(2311)$ & $5243(2268)$ & $5304(2460)$ & $1801(-)$ & $4575(-)$ & 5249 (5539) \\
\hline$-E$ & $5554(2498)$ & $5498(2516)$ & $5591(2595)$ & $2685(77)$ & $5541(368)$ & $5719(3754)$ \\
\hline \multicolumn{7}{|c|}{ No cisgénero } \\
\hline & $2 \quad 2$ & $\sqrt{\text { ivt }}$ & & & & \\
\hline TR & $M(D E)$ & $M(D E)$ & $M(D E)$ & $M(D E)$ & $M(D E)$ & $M(D E)$ \\
\hline$-\mathrm{O}$ & $5532(1372)$ & $5024(1448)$ & $5215(2224)$ & $4110(1712)$ & $5627(3900)$ & 4885 (2346) \\
\hline$-X$ & 4890 (1901) & $4900(2642)$ & $4444(1637)$ & - & $6984(-)$ & $5061(3776)$ \\
\hline$-E$ & $5712(2793)$ & $5298(2240)$ & $4769(1865)$ & - & - & - \\
\hline
\end{tabular}

Teniendo en cuenta las hipótesis experimentales, se realizó un LMM con Morfología e Identidad de género como efectos fijos y Estereotipicidad y Tipo de respuesta anidados a la Morfología. Participantes e Ítems se sumaron al modelo como efectos aleatorios. En la Tabla 6 se reporta el modelo realizado.

Tabla 6 - Reporte del LMM 


\begin{tabular}{|c|c|c|c|c|}
\hline \multicolumn{5}{|c|}{ Efectos fijos } \\
\hline & Est/Beta & Error estándar & $t$ & $p$ \\
\hline Intercepto & 8.55328 & 0.02583 & 331.147 & $<2 \mathrm{e}-16 * * *$ \\
\hline Morfología_X-O & -0.15133 & 0.04752 & -3.185 & $0.00146 * *$ \\
\hline Morfología_E-X & 0.08460 & 0.06330 & 1.337 & 0.18143 \\
\hline Id. género_Varones-Mujeres & 0.01405 & 0.02891 & 0.486 & 0.62721 \\
\hline Morfología_O:Estereotipicidad_Media-Baja & -0.05422 & 0.02700 & -2.008 & $0.04968 *$ \\
\hline Morfología_X:Estereotipicidad_Media-Baja & -0.11135 & 0.13300 & -0.837 & 0.40250 \\
\hline Morfología_E:Estereotipicidad_Media-Baja & 0.24803 & 0.12173 & 2.038 & $0.04164 *$ \\
\hline Morfología_O:Estereotipicidad_Alta-Media & 0.04921 & 0.02685 & 1.833 & 0.07255 \\
\hline Morfología_X:Estereotipicidad_Alta-Media & 0.08183 & 0.08476 & 0.965 & 0.33437 \\
\hline 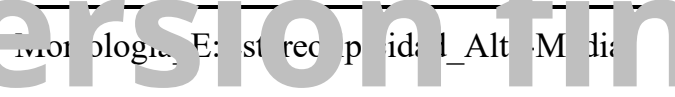 & $\overline{0 .} \overline{2044}$ & v. 79 & & $5.0 \overline{44}$ \\
\hline Morfología_O:Est_Baja:RTA_Varones-Mixto & -0.07574 & 0.03272 & -2.315 & $0.02062 *$ \\
\hline Morfología_X:Est_Baja:RTA_Varones-Mixto & 0.01634 & 0.21530 & 0.076 & 0.93951 \\
\hline Morfología_E:Est_Baja:RTA_Varones-Mixto & -0.17557 & 0.17182 & -1.022 & 0.30689 \\
\hline $\begin{array}{l}\text { Morfología_O:Est_Media:RTA_Varones- } \\
\text { Mixto }\end{array}$ & -0.19544 & 0.03069 & -6.368 & $2.01 \mathrm{e}-10 * * *$ \\
\hline $\begin{array}{l}\text { Morfología_X:Est_Media:RTA_Varones- } \\
\text { Mixto }\end{array}$ & -0.18820 & 0.15305 & -1.230 & 0.21887 \\
\hline $\begin{array}{l}\text { Morfología_E:Est_Media:RTA_Varones- } \\
\text { Mixto }\end{array}$ & 0.37172 & 0.17105 & 2.173 & $0.02979 *$ \\
\hline Morfología_O:Est_Alta:RTA_Varones-Mixto & -0.33894 & 0.03223 & -10.515 & $<2 \mathrm{e}-16 * * *$ \\
\hline Morfología_X:Est_Alta:RTA_Varones-Mixto & -0.11716 & 0.06936 & -1.689 & 0.09123 \\
\hline Morfología_E:Est_Alta:RTA_Varones-Mixto & -0.07431 & 0.09498 & -0.782 & 0.43399 \\
\hline \multicolumn{5}{|c|}{ Efectos aleatorios } \\
\hline
\end{tabular}




\begin{tabular}{|l|c|c|}
\hline & Varianza & DE \\
\hline Participantes (intercepto) & 0.0682782 & 0.26130 \\
\hline Ítems (intercepto) & 0.0008089 & 0.02844 \\
\hline \multicolumn{2}{|c|}{ Ajuste del modelo } \\
\hline $\mathrm{R}^{2}$ & Marginal & Condicional \\
\hline \multicolumn{1}{|c|}{0.02281334} & 0.2570331 \\
\hline $\begin{array}{l}\text { Los } p \text {-valor de los efectos fijos fueron calculados con las aproximaciones de Satterthwaite. } \\
\text { Ecuación del modelo: log(TRrespuesta) (Morfología / Estereotipicidad / RTA) + Identidad de género + (1 | Participantes) } \\
+(1 \mid \text { Ítems) }\end{array}$ & \\
\hline
\end{tabular}

RTA= Tipo de respuesta

En el modelo reportado (Tabla 6), se encontró un efecto principal del factor Morfología a favor de las variantes morfológicas no binarias $(p=0.00146)$. No se encontraron diferencias estadísticamente significativas entre ambas variantes no binarias $(p=0.18143)$. No se encontró un efecto prir.ipal de la Io mindad de génerc de les participantes $(p=0.62721)^{9}$. En cuanto a la stere ti ic da, e c ıcon ó $\mathrm{n}$ fe to ar di lo a ra M rtolo ía en $\mathrm{f}$ rt u ar 1 rra lá va ianı morfológica [-e]. En relación con el Tipo de respuesta, se encontraron diferencias estadísticamente significativas anidadas al masculino genérico para todos los niveles de Estereotipicidad.

Por último, corresponde mencionar que, debido a que solicitamos diversos datos demográficos, pudimos indagar sobre posibles efectos del nivel de escolarización formal de les participantes: no se hallaron efectos significativos en función de este factor. Respecto de la edad, en cambio, la muestra era diversa. Esto, por un lado, evitó proyectar resultados anómalos que suelen surgir de muestras WEIRD (HENRICH; HEINE; NORENZAYAN, 2010), pero, por el otro, no permitió generar subgrupos homogéneos y equilibrados para realizar contrastes adecuados respecto de esta variable. Proyectamos concentrarnos sobre este factor en próximos experimentos considerando Edad como parte del diseño experimental inicial.

\footnotetext{
${ }^{9}$ Se probó correr un modelo estadístico más complejo con los demás factores anidados a la Identidad de género, pero dicho modelo no convergía. Sin embargo, se hizo un análisis segmentado y se encontró que hubo diferencias estadísticamente significativas entre varones y mujeres sólo para el tipo de respuesta frente al masculino genérico y estereotipicidad baja. Como se ve en la Figura 4, las mujeres tardaron más en asignar una referencia mixta al masculino genérico de estereotipicidad baja.
} 


\section{Discusión}

A diferencia de la primera tarea, este segundo experimento muestra procesos automáticos durante el procesamiento del lenguaje y su relación con las representaciones de género "por defecto", implícitas o no conscientes.

En principio, es interesante notar la distribución del tipo de respuestas. Tal como mencionamos en el apartado de Resultados, el primer dato a tener en cuenta es la modulación que ejerce la Estereotipicidad de los nombres de rol sobre las elecciones de posibles referentes cuando el sintagma se presenta con la forma [-o], es decir, el masculino genérico. Modulación que no ocurre para ninguna de las dos formas morfológicas no binarias. En consecuencia, podemos destacar que las formas no binarias parecen funcionar como formas lingüísticas y semánticas más precisas e inequívocas respecto de su capacidad referencial: sin importar si los nombres de rol están más o menos asociados a una determinada identidad sexo-genérica, cualquiera de las formas no binarias parece generar de modo consistente representaciones de grupos de personas sin género uniforme. Este no es, sin embargo, el mismo cuadro que se da para los sintagmas nominales con la forma de masculino genér :o; en este caso parece haher una renresentación lixta ó, ar $1 \mathrm{~S} 1 \mathrm{jmb}$ is e ol q e o stén fuer smen e ;ocic lo a pri ri ce la gun identidad sexo-genérica o estereotipo de género. Dicho de otro modo, en caso de querer referirnos a grupos mixtos de personas cuya profesión sea, por ejemplo, la plomería, usar la forma morfológica de masculino genérico no lograría generar esa representación mixta en la interpretación de les hablantes. En cambio, utilizar cualquiera de las formas no binarias sí lograría generar representaciones de grupos mixtos.

En segunda instancia, es preciso analizar los resultados vinculados con el proceso online de selección de respuesta, es decir, el tiempo que toma hacer la elección de una opción para cada referencia. En este sentido, la interpretación de esos datos supone entender el tiempo de respuesta como un reflejo del proceso que suyace a la construcción o recuperación de una representación mental sexo-genérica adecuada para cada sintagma nominal que permita generar una referencia válida.

En esta línea, un primer punto que es preciso destacar es que el factor Morfología generó un efecto principal en los tiempos de respuesta, pero en sentido inverso al que, en principio, se podía hipotetizar y distinto al patrón exhibido por los juicios de aceptabilidad en el Experimento 1: los sintagmas con masculino genérico requirieron tiempos de respuesta mayores que aquellos con cualquiera de las dos variantes morfológicas no binarias. Dentro de las variantes no binarias 
no se hallaron diferencias estadísticamente significativas; es decir que el masculino genérico se distingue de las formas no binarias, pero, al menos en texto escrito, las variantes [-x] y [-e] no muestran diferencias en la velocidad requerida para manipular una representación mental y generar una referencia hacia grupos mixtos o con género no uniforme. Es importante notar que este patrón interno a las formas no binarias es similar al hallado para juicios de aceptabilidad, aunque es necesario desarrollar estudios que indaguen sobre el procesamiento de enunciados orales y de formas que requieran cambios ortográficos, ya que allí podría ubicarse la mayor diferencia entre estas dos variantes.

Si analizamos los tiempos de respuesta en cada una de las tres variantes morfológicas, notamos que las mujeres que eligen una referencia hacia grupos mixtos a partir de un sintagma nominal de estereotipicidad baja con masculino genérico tardan más en hacer esa elección que los varones. Sin embargo, para ambos grupos de participantes existe un patrón similar cuando se compara la elección de referencia mixta para sintagmas con masculino genérico respecto de la referencia hacia grupos de varones: en los casos en que la morfología de genérico es ambigua, responder por una opción mixta toma más tiempo, es decir, exhibe un mayor costo de procesamiento. Esta misma interpretación se verá respaldada por un efecto similar que surge a partir de id in Idencia del $f$ ctor Estereotinic lad, que comentaremos más adelante.

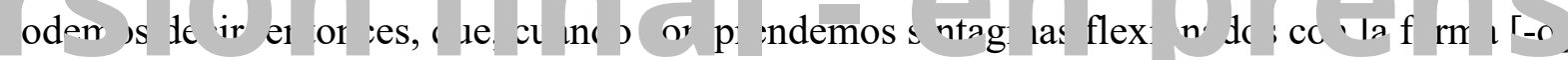
de masculino genérico, construir una referencia hacia grupos constituidos por personas de diversos géneros es no sólo más costoso en términos del resultado final de dicha referencia tipo de respuesta, precisión en la referencia mixta- sino también en virtud del proceso subyacente -los tiempos de elección son mayores-. Ambas medidas en coordinación construyen un patrón en el que no sólo la referencia que habilitan las variantes no binarias parece ser inequívoca y consistente hacia grupos mixtos, sino que además, los tiempos requeridos para ese proceso son más breves para esos casos de referencias no ambiguas que para los que supone el masculino genérico como referencia ambigua entre grupos exclusivos de varones o grupos mixtos, en los que ambas representaciones podrían estar compitiendo.

Asimismo, vale mencionar que la Estereotipicidad resultó un efecto modulador del proceso cuando se lo analiza en función del tipo de respuesta. En líneas generales, el nivel medio de Estereotipicidad no se diferencia significativamente de los otros dos niveles, por lo que parece haber respaldo para considerar que ese nivel medio pueda estar exhibiendo un proceso diacrónico y paulatino de mayor uso de formas no binarias para esos sintagmas, es decir, una suerte de efecto de frecuencia de exposición a sintagmas como funcionarixs en su forma no binaria - una frecuencia, en este corte sincrónico, menor a la de, por ejemplo, niñxs-. Existe, 
además, un efecto del Tipo de Respuesta anidado a la variante morfológica [-o] para los tres niveles de Estereotipicidad: la referencia mixta parece generar un mayor costo de procesamiento, con tiempos de respuesta significativamente mayores, respecto de las respuestas que exhiben una representación que construye una referencia exclusiva de varones. Podemos interpretar, entonces, que para nombres de rol con asociaciones sexo-genéricas fuertes, las formas en masculino genérico producen un primer sesgo por defecto hacia la interpretación masculina exclusiva, y llegar a una interpretación mixta quizá suponga una segunda etapa en el procesamiento, con intervención de posibles revisiones o monitoreos.

Por último, queremos centrarnos en el análisis de la Identidad de género de les participantes. Si bien en los dos grupos identificados como cis género, el masculino genérico muestra mayores tiempos de respuesta, el patrón general que se muestra en la Figura 4 evidencia algunas particularidades para cada caso. Sólo 21 participantes se identificaron como no cis género -un número insuficiente para obtener la mínima variabilidad necesaria para constituir una muestra representativa-, y sólo ese grupo muestra un patrón de respuestas y tiempos de respuesta diferente. Cuando analizamos los modelos sólo con dos niveles de identidad de género -varón cis y mujer cis-, notamos que no hay diferencias significativas debidas a ese factor y el patrón de tiempos de respuesta no vana en función r a la Identidad de género de les participantes. Esto s: di a te $n x$ sc fur te le te a $x$ ac is a nive es c roce ar ie to alitol át sc manipulación no consciente o implícita de representaciones mentales, el proceso psicolingüístico subyacente que exhiben mujeres y varones es similar, más allá de que en tareas estratégicas, mediadas por las ideologías lingüísticas y las representaciones sexo-genéricas explícitas puedan existir diferencias en función de este factor. Este parece ser uno de los puntos clave para analizar las diferencias debidas a la tarea, fenómeno que retomaremos en la Discusión general.

\section{Discusión general}

Tal como mencionamos en la Introducción, no existen trabajos que analicen el procesamiento psicolingüístico de formas no binarias en español. Si bien existen algunos estudios sobre percepciones, creencias y evaluación de usos lingüísticos sin sesgo de género en español y otras lenguas (JIMÉNEZ RODRIGO; ONSALO; TRAVERSO CORTÉS, 2011; KAUFMANN; BOHNER, 2014; LEAPER, 2014; PREWITT-FREILINO; CASWELL; LAAKSO, 2012), 
tampoco existían estudios que indagaran estrictamente sobre los niveles de aceptabilidad que tienen frases concretas usadas con las variantes morfológicas no binarias.

A partir de este trabajo, nos interesa no sólo comenzar a estudiar estos fenómenos con mayor sistematicidad, sino también analizar las diferencias que pueden surgir en los resultados dependiendo de la tarea, o mejor dicho, del proceso subyacente que supone la realización de cada una de las tareas.

Por un lado, en la tarea de juicios de aceptabilidad, lo que se evalúa es el grado de aceptación consciente que les hablantes de una comunidad lingüística muestran, en este caso, sobre sintagmas nominales que se presentan con variantes de morfología de género que no corresponden al paradigma binario del español (-o y -a). Esta tarea, en ese sentido, supone una tarea de decisión voluntaria, sobre la que operan una cantidad de creencias; no sólo representaciones y estereotipos de género sino también ideologías lingüísticas y matrices discursivas dominantes, que construyen, a su vez, marcos de sentido común dominante (MORENO CABRERA, 2008; PÉREZ; MORAGAS, 2020; SAYAGO, 2019). Así es como una tarea de juicios de aceptabilidad pone en juego no sólo procesos y representaciones mentales de modo implícito, sino que involucra muchos de estos factores, además de las normas v prescrnciones lingüísti as impuestas pc instituciones formales más allá de los usos ngïis ic s al th lle y ce id in s se se la lantes.

Una tarea de comprensión de oraciones en la que se miden no sólo el tıpo de respuestas sino los tiempos requeridos para llevar adelante la comprensión, en cambio, apunta a estudiar procesos psicolingüísticos automáticos, muchos de ellos fuera del control ejecutivo y al margen de los juicios conscientes que puedan generar les hablantes para o durante la realización de la tarea. Es así como, de este modo, es posible evaluar representaciones manipuladas cognitivamente de modo implícito cuando procesamos lenguaje: más allá de si creemos que una variante morfológica es más o menos válida para el español, si podemos lograr comprenderla de modo adecuado y sin elevados costos de procesamiento, tenemos datos de respaldo para refutar que existan obstáculos estrictamente (psico)lingüísticos que atenten contra su uso o su adecuada comprensión.

Ante este marco general, los resultados reportados en este trabajo resultan especialmente relevantes. Los dos experimentos presentados muestran, individualmente, datos novedosos sobre el uso y la comprensión de variantes morfológicas no binarias en español y esos hallazgos se han discutido en los apartados correspondientes. En esta última sección, nos interesa poner en foco las diferencias interesantes que hemos encontrado debidas a la tarea y al proceso subyacente que cada una impone. Como pudimos ver en el Experimento 1, las variantes no 
binarias (-x y -e) siguen generando una menor aceptación que el masculino genérico por parte de les hablantes. Sobre todo los varones muestran más rechazo hacia formas no binarias y, en general, ese rechazo se observa sobre los nombres de rol que tienen un sesgo de género muy marcado por estar asociados a estereotipos fuertes -i.e. plomeros-. Sin embargo, cuando lo que se solicita a le hablante no es un juicio sino que comprenda una oración e indique la referencia de un sintagma nominal, el patrón de resultados cambia: las variantes no binarias son más precisas -no ofrecen ambigüedad sobre su referencia-y se procesan sin costos cognitivos extra respecto del masculino genérico. Es decir que cuando están en juego creencias conscientes sobre representaciones de género e ideologías lingüísticas, les hablantes exhiben posicionamientos explícitos muy distintos -incluso en algunos casos opuestos- que cuando procesan lenguaje, sin que este proceso esté bajo su control ejecutivo y consciente.

Existe, no obstante, un punto de contacto interesante entre los resultados hallados en las dos tareas. Los nombres de rol con estereotipicidad alta se comportan siempre de un modo particular: así como las formas no binarias son menos aceptadas en estos casos, el procesamiento de esos sintagmas cuando se presentan en la condición de masculino genérico produce un sesgo importante hacia una referencia exclusiva de varones, y la referencia mixta sólo se ve hakılitada consi entemente cuand se presentan en las condiciones con las variantes

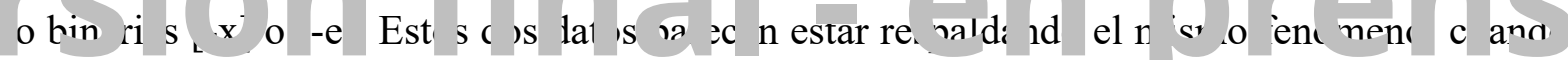
existe un sesgo de género muy fuerte sobre las referencias posibles de un nombre de rol, existen, al menos dos proyecciones. Por un lado, resulta menos frecuente y, por ende, menos aceptado un sintagma que marque género no binario en esos sustantivos -i.e. plomeres-; pero, simultáneamente, la ausencia de esa marca invisibiliza la posibilidad de que dentro de un grupo de personas que ejercen la plomería haya, por ejemplo, mujeres plomeras. Esto nos pone ante la disyuntiva paradójica clásica en muchos estudios que analizan la relación entre pensamiento y lenguaje. Qué es primero: no existen muchas mujeres plomeras entonces las personas, al leer los plomeros sesgan sus representaciones hacia varones exclusivamente -mucho más que con los niños-o, viceversa, al no poder nombrar precisamente con un sintagma genérico a personas que ejerzan la plomería sin distinción de género se construye una representación inadecuada de que sólo existen varones plomeros y se invisibiliza la existencia de una parte de ese grupo. Dicho de otro modo, si comenzáramos a usar plomeres como forma de genérico que no aporta ambigüedad sobre que las personas que conforman ese grupo no se identifican todas con el género masculino, ¿podríamos comenzar a generar representaciones de plomeras mujeres habilitando la posibilidad de deconstruir y desnaturalizar estereotipos categóricos? 
Los datos analizados en este trabajo están en línea con estudios realizados en otras lenguas (KAUFMANN; BOHNER, 2014; LEAPER, 2014; SATO; GYGAX; GABRIEL, 2016; SCZESNY; MOSER; WOOD, 2015; VIGLIOCCO et al. 2005) respecto de los sesgos consistentes y estables que pueden proyectarse desde el uso de determinadas formas lingüísticas hacia las representaciones mentales que manejamos sobre el mundo y las relaciones que en él se dan. En este caso, los datos en español también indican que la variante morfológica conocida como masculino genérico, que, en teoría lograría referir y generar representaciones de grupos con género no uniforme, no funciona estrictamente así y, en cambio, genera sesgos respecto de esa referencia fuertemente dependientes de los estereotipos de género vinculados a los nombres de rol. Pero, además, logramos mostrar que las variantes morfológicas no binarias no implican mayores costos de procesamiento u obstáculos en la comprensión, al contrario, resultan formas más precisas que el masculino genérico para nombrar, referir y representar la diversidad de géneros que puede hallarse dentro de un grupo de personas.

Sin ánimo de cerrar la pregunta sobre "qué es primero" con una respuesta categórica, nuestros datos muestran que el uso de formas no binarias puede lograr visibilizar adecuadamente esta diversidad, al tiempo que exhibe que las barreras para su uso no provienen estrictamente del sistema ac la tengua ni de is mecanismos cr snitivos que subyacen a su procesamiento.

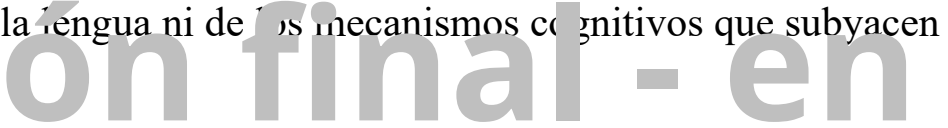

BINARY OR NON-BINARY? GENDER MORPHOLOGY IN SPANISH: DIFFERENCES DEPENDENT ON THE TASK

\begin{abstract}
There is empirical evidence in different languages on how the computation of gender morphology during psycholinguistic processing affects the conformation of sex-generic representations. However, there is no empirical evidence on the processing of non-binary morphological variants in Spanish (-x or -e) in contrast to the generic masculine variant (-o). To analyze this phenomenon, we conducted two experiments: an acceptability judgment task and a sentence comprehension task. The results show differences depending on the task. This means that the underlying processes put into play in each one generate different effects. In acceptability judgments, which involve strategic processes mediated by beliefs and the linguistic norm, the generic masculine is more acceptable to refer to mixed groups. In the sentence comprehension task, which inquires about automatic processes and implicit representations, the non-binary forms consistently elicited a reference to mixed groups. Furthermore, the response times indicated that these morphological variants do not entail a higher processing cost than the generic masculine.
\end{abstract}


KEYWORDS: Psycholinguistics. Gender. Morphology. Gender stereotypes.

\section{REFERENCIAS}

BAAYEN, H.; MILIN, P. Analyzing Reaction Times. International Journal of Psychological Research, Medellín, v.3, n.2, p.12-28, 2010.

BARR, D. J.; LEVY, R.; SCHEEPERS, C.; TILY, H. J. Random effects structure for confirmatory hypothesis testing: Keep it maximal. Journal of Memory and Language, Cambridge, v.68, n.3, p.255-278, 2013.

BARTÓN, K. MuMIn: Multi-Model Inference. R package version 1.43.17. 2020. Disponible en: $<$ https://CRAN.R-project.org/package=MuMIn>. Consultado en: 23 nov. 2020.

BATES, D.; MAECHLER, M.; BOLKER, B.; WALKER, S. Fitting Linear Mixed-Effects Models Using lme4. Journal of Statistical Software, Los Angeles, v.67, n. 1, p.1-48, 2015.

BRADLEY, E.D. The influence of linguistic and social attitudes on grammaticality judgments of singular 'they'. Language Sciences, Amsterdam, v.78, 101272, 2020. doi: 10.1016/j.langsci.2020.101272

BRAUN, F.; SCZESNY, S.; STAHLBERG, D. Cognitive effects of masculine generics in German: An overview of empirical findings. Communications, Berlin, v.30, n. 1, p.1-21, 2005.

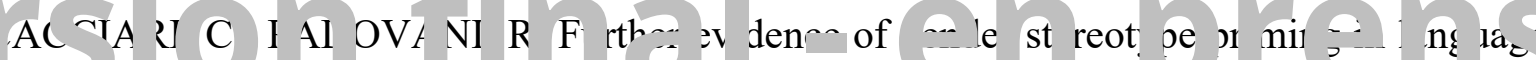

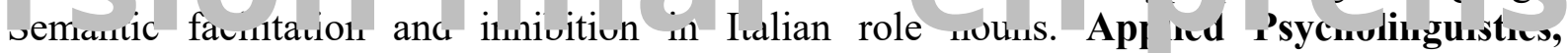
Cambridge, v.28, p.277-293, 2007.

CORBETT, G. G. Gender. Cambridge: Cambridge University Press, 1991.

ENDERSEN, A.; JANDA, L. A. Five statistical models for Likert-type experimental data on acceptability judgments. 2015. Disponible en: <https://ninum.uit.no/handle/10037/8007>. Consultado en: 23 nov. 2020.

COUSINEAU, D.; CHARTIER, S. Outliers Detection and Treatment: A review. International Journal of Psychological Research, Medellín, v.3, n.1, p.58-67, 2010.

DIXON, J. The question of genres. En REID, I. (Ed). The place of genre in learning: Current debates. Victoria: Typereader Publications, 1987, p.9-21.

EVERETT, G. Linguistic relativity: Evidence across languages and cognitive domains. Berlín: De Gruyter Mouton, 2013.

FLAHERTY, M. How a language gender system creeps into perception. Cross-cultural Psychology, New York, v.32, n.1, p.18-31, 2001.

GYGAX, P. M.; ELMINGER, D.; ZUFFEREY, S.; GARNHAN, A; SCZESNY, S.; VON STOCKHAUSEN, L.; BRAUN, F.; OAKHILL, J. A language index of grammatical gender 
dimensions to study the impact of grammatical gender on the way we perceive women and men. Frontiers in Psychology, Lausanne, v.10, 1604, 2019. doi: 10.3389/fpsyg.2019.01604

GYGAX, P.; GABRIEL, U. Can a group of musicians be composed of women? Generic interpretation of French masculine role names in the absence and presence of feminine forms. Swiss Journal of Psychology, Bern, v.67, n.3, p.143-151, 2008.

GYGAX, P.; GABRIEL, U.; SARRASIN, O.; OAKHILL, J.; GARNHAM, A. Generically intended, but specifically interpreted: When beauticians, musicians, and mechanics are all men. Language and Cognitive Processes, Oxon, v.23, n.3, p.464-485, 2008.

HENRICH, J.; HEINE, S.; NORENZAYAN, A. The weirdest people in the world? Behavioral and Brain Sciences, 33(2-3), p. 61-83, 2010. doi:10.1017/S0140525X0999152X

IMAI, M.; SCHALK, L.; SAALBACH, H.; OKADA, H. All giraffes have female-specific properties: Influence of grammatical gender on deductive reasoning about sex-specific properties in German speakers. Cognitive Science: A Multidisciplinary Journal, Seattle, v.38, p.514-536, 2014.

JIMÉNEZ RODRIGO, M.L.; ONSALO, M.L.; TRAVERSO CORTÉS, J. Lenguaje no sexista y barreras a su utilización. Un estudio en el ámbito universitario. Revista de Investigación en Educación, Vigo, v.9, n.2, p.174-183, 2011.

KAUFMANN, C.; BOHNER, G. Masculine generics and gender-aware alternatives in Spanish. IZGOnZeit. Interdisziplin ren Zentrums f $\mathbf{r}$ Geschlechterforschung (IZG), Bielefeld, p.8$1,2 ? 4$

KONISHI, T. The semantics of grammatical gender: A cross-cultural study. Journal of Psycholinguistic Research, New York, v.22, p.519-534, 1993.

KUZNETSOVA, A.; BROCKHOFF, P. B.; CHRISTENSEN, R. H. B. LmerTest Package: Tests in Linear Mixed Effects Models. Journal of Statistical Software, Los Angeles, v.82, n.13, p.1-26, 2017.

LEAPER, C. Gender similarities and differences in language. En HOLTGRAVES, T. M. (Ed). The Oxford handbook of language and social psychology. Oxford: Oxford University Press, 2014.

LUCY, J. A. The scope of linguistic relativity: An analysis and review of empirical research. En GUMPERZ, J. J.; LEVINSON, S. C. (Eds). Rethinking linguistic relativity. Cambridge: Cambridge University Press, 1996, p.37-69.

MACIUSZEK, J.; POLAK, M.; ŚWIĄTKOWSKA, N. Grammatical gender influences semantic categorization and implicit cognition in Polish. Frontiers in Psychology, Lausanne, v.10, 2208, 2019. doi: 10.3389/fpsyg.2019.02208

MISERSKY, J.; MAJID, A.; SNIJDERS, T. M. Grammatical gender in German influences how role-nouns are interpreted: Evidence from ERPs. Discourse Processes, New York, v.56, n.8, p.643-654, 2019. 
MORENO CABRERA, J.C. Gramáticos y academias. Para una sociología del conocimiento de las lenguas. Arbor. Ciencia, pensamiento y cultura, Madrid, v. 731, 519-528, 2008.

PÉREZ, S.I.; MORAGAS, F. Lenguaje inclusivo: malestares y resistencias en el discurso conservador. En KALINOWSKI, S.; GASPARI, J.; PÉREZ, S.I.; MORAGAS, F. Apuntes sobre lenguaje no sexista e inclusivo. Rosario: UNR Editora, 2020, p.69-96.

PINKER, S. El instinto del lenguaje. Madrid: Alianza, 1999.

PRENTICE, D. A. Do language reforms change our way of thinking? Journal of Language and Social Psychology, New York, v.13, n.1, p.3-19, 1994.

PREWITT-FREILINO, J.L.; CASWELL, T.A.; LAAKSO, E.K. The gendering of language: a comparison of gender equality in countries with gendered, natural gender, and genderless languages. Sex Roles, New York, v.66, p.268-281, 2012.

R CORE TEAM. R: A language and environment for statistical computing. Vienna: R Foundation for Statistical Computing, 2021. Disponible en <https://www.R-project.org/>. Consultado en: 9 dic. 2021.

RATCLIFF, R. Methods with Dealing with Reaction Time Outliers. Psychological Bulletin, Washington, v.114, n.3, p.510-532, 1993.

SAALBACH, H.; IMAI, M.; SCHALK, L. Grammatical gender and inferences about biological properties in German-spe: uris children. Cr gnitive Science: A Multidisciplinary Journal, eat. $v 31 \quad \mathrm{p} / 21 \cdot 267202$

SAPIR, E. An introduction to the study of speech. New York: Harcourt, Brace, 1921.

SATO, A.; GYGAX, P.; GABRIEL, U. Gauging the Impact of Gender Grammaticization in Different Languages: Application of a Linguistic-Visual Paradigm. Frontiers in Psychology, Lausanne, v.7, 140, 2016. doi: 10.3389/fpsyg.2016.00140

SAYAGO, S. Apuntes sociolingüísticos sobre el lenguaje inclusivo. Revista científica de la red de carreras de Comunicación Social, n.9, 2019. doi: 10.24215/24517836e015

SCHAD, D. J.; VASISHTH, S.; HOHENSTEIN, S.; KLIEGL, R. How to capitalize on a priori contrasts in linear (mixed) models: A tutorial. Journal of Memory and Language, Cambridge, v.110, n.104038, 2020.

SCZESNY, S.; MOSER, F.; WOOD, W. Beyond Sexist Beliefs: How Do People Decide to Use Gender-Inclusive Language? Personality and Social Psychology Bulletin, New York, v.41, n. 7, p.943-954, 2015.

SCOTTO, S.C.; PÉREZ, D.I. Relatividad lingüística, gramáticas de género y lenguaje inclusivo: algunas consideraciones. Análisis Filosófico, Buenos Aires, v.40, n.1, p.5-39, 2020.

SEGEL, E.; BORODITSKY, L. Grammar in art. Frontiers in Psychology, Lausanne, v.1, 244, 2011. doi: 10.3389/fpsyg.2010.00244 
SERA, M.; ELIEF, C.; FORBES, J.; BURCH, M. C.; RODRIGUEZ, W.; DUBOIS, D. P. When language affects cognition and when it does not: an analysis of grammatical gender and classification. Journal of Experimental Psychology: General, Washington, v.131, p.377397, 2002.

SLOBIN, D. Learning to think for speaking: Native language, cognition, and rhetorical style. Pragmatics, Amsterdam, v.1, n.1, p.7-25, 1991.

SLOBIN, D. From 'Thought and Language' to 'Thinking for Speaking'. En LEVINSON, S. C.; GUMPERZ, J. J. (Eds). Rethinking linguistic relativity. Cambridge: Cambridge University Press, 1996, p.70-96.

STAHLBERG, D.; BRAUN, F.; IRMEN, L.; SCZESNY, S. Representation of the sexes in language. En FIEDLER, K. (Ed). Frontiers of social psychology. Social communication. New York: Psychology Press, 2007, p.163-187.

VIGLIOCCO, G.; VINSON, D.; PAGANELLI, F.; DWORZYNSKI, K. Grammatical Gender Effects on Cognition: Implications for Language Learning and Language Use. Journal of Experimental Psychology: General, Washington, v.134, p.501-520, 2005.

WHORF, B. L. Language, thought and reality. New York: The MIT Press, 1956.

ZEHR, J.; SCHWARZ, F. PennController for Internet Based Experiments (IBEX). 2018. doi: 10.17605/OSF.IO/MD832

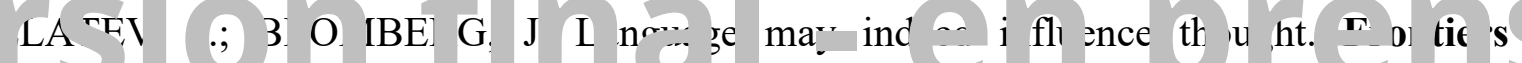
psycrolozy, Lausanrid, v.6, 1631, 2615. doi: $10.3389 /$ fpsyg.2J15.016

ZUNINO, G. M.; STETIE, N. A. Procesamiento de formas no binarias en español: relación entre el uso voluntario y la comprensión. En evaluación. 